\title{
$v_{1}$-PERIODIC HOMOTOPY GROUPS OF EXCEPTIONAL LIE GROUPS: TORSION-FREE CASES
}

\author{
MARTIN BENDERSKY, DONALD M. DAVIS, AND MAMORU MIMURA
}

\begin{abstract}
The $v_{1}$-periodic homotopy groups $v_{1}^{-1} \pi_{*}(X ; p)$ are computed explicitly for all pairs $(X, p)$, where $X$ is an exceptional Lie group whose integral homology has no $p$-torsion. This yields new lower bounds for $p$-exponents of actual homotopy groups of these spaces. Delicate calculations with the unstable Novikov spectral sequence are required in the proof.
\end{abstract}

\section{Statement of Results}

If $p$ is prime, the $p$-local $v_{1}$-periodic homotopy groups $v_{1}^{-1} \pi_{*}(X ; p)$ can be defined, similarly to [13], for any space $X$ which has an eventual $H$-space exponent at $p$. We review this definition at the end of this section. These groups are interesting because they are often almost completely computable and yet they give large direct summands of actual homotopy groups of $X$. Using results of [17] and [11], we show at the end of this section that all compact Lie groups have such exponents for all $p$, and so we should try to compute their $v_{1}$ periodic homotopy groups. This was done at the odd primes for $\mathrm{SU}(n), \mathrm{Sp}(n)$, and $\mathrm{SO}(n)$ in [13], for $\mathrm{SU}(n)$ at the prime 2 in [8], and for $G_{2}$ at the prime 2 in [14]. In this paper we do it for all exceptional Lie groups $X$ at all primes $p$ for which $H_{*}(X ; \mathbf{Z})$ has no $p$-torsion. In the process, we also calculate the $v_{1}$ periodic homotopy groups of many sphere bundles over spheres. We also read off some results about $p$-exponents of the exceptional Lie groups; in particular, we show that they are not quite independent of the prime $p$.

Our results depend heavily upon the following splitting theorem of $[23,21$, 27] for the $p$-localizations of the exceptional Lie groups. That these are the only torsion-free cases was established in [10].

Theorem 1.1. The exceptional Lie groups when localized at $p$ split as indicated below.

Received by the editors July 27, 1990.

1980 Mathematics Subject Classification (1985 Revision). Primary 57T20.

Key words and phrases. Exceptional Lie groups, periodic homotopy groups, unstable Novikov spectral sequence.

The first two authors were partially supported by the National Science Foundation. The first and third authors would like to thank MSRI, where this work was begun. 


$$
\begin{aligned}
& G_{2} \quad p=3 \quad B_{2}(3,11) \\
& p=5 \quad B(3,11) \\
& p>5 \quad S^{3} \times S^{11} \\
& F_{4} \quad p=5 \quad B(3,11) \times B(15,23) \\
& p=7 \quad B(3,15) \times B(11,23) \\
& p=11 \quad B(3,23) \times S^{11} \times S^{15} \\
& p>11 S^{3} \times S^{11} \times S^{15} \times S^{23} \\
& E_{6} \quad p=5 \quad F_{4} \times B(9,17) \\
& p>5 \quad F_{4} \times S^{9} \times S^{17} \\
& E_{7} \quad p=5 \quad B(3,11,19,27,35) \times B(15,23) \\
& p=7 \quad B(3,15,27) \times B(11,23,35) \times S^{19} \\
& p=11 \quad B(3,23) \times B(15,35) \times S^{11} \times S^{19} \times S^{27} \\
& p=13 \quad B(3,27) \times B(11,35) \times S^{15} \times S^{19} \times S^{23} \\
& p=17 \quad B(3,35) \times S^{11} \times S^{15} \times S^{19} \times S^{23} \times S^{27} \\
& p>17 S^{3} \times S^{11} \times S^{15} \times S^{19} \times S^{23} \times S^{27} \times S^{35} \\
& E_{8} \quad p=7 \quad B(3,15,27,39) \times B(23,35,47,59) \\
& p=11 \quad B(3,23) \times B(15,35) \times B(27,47) \times B(39,59) \\
& p=13 \quad B(3,27) \times B(15,39) \times B(23,47) \times B(35,59) \\
& p=17 \quad B(3,35) \times B(15,47) \times B(27,59) \times S^{23} \times S^{39} \\
& p=19 \quad B(3,39) \times B(23,59) \times S^{15} \times S^{27} \times S^{35} \times S^{47} \\
& p=23 \quad B(3,47) \times B(15,59) \times S^{23} \times S^{27} \times S^{35} \times S^{39} \\
& p=29 \quad B(3,59) \times S^{15} \times S^{23} \times S^{27} \times S^{35} \times S^{39} \times S^{47} \\
& p>29 S^{3} \times S^{15} \times S^{23} \times S^{27} \times S^{35} \times S^{39} \times S^{47} \times S^{59}
\end{aligned}
$$

Here each space $B\left(2 n_{1}+1, \ldots, 2 n_{r}+1\right)$ is built up from fibrations involving p-local spheres of the indicated dimensions, and is equivalent to a direct factor of the p-localization of $\mathrm{SU}\left(n_{r}+1\right) / \mathrm{SU}\left(n_{1}\right)$. The attaching map of the middle cell of $B(2 n+1,2 n+2 p-1)$ is $\alpha_{1} \in \pi_{2 n+2 p-2}\left(S^{2 n+1}\right)$. The only factor not of this type, labeled $B_{2}(3,11)$, is a sphere bundle with attaching map $\alpha_{2}$, and is not a direct factor in the 3-localization of a quotient of SU's.

Here $\alpha_{t}$ denotes the standard element of order $p$ in $\pi_{2(p-1) t-1}\left(S^{0}\right)$ (see, e.g., $[24,1.3 .11])$. Of course, the $v_{1}$-periodic homotopy groups of a product space are just the product of the $v_{1}$-periodic homotopy groups of the factors, and so the $v_{1}$-periodic homotopy groups of all torsion-free exceptional Lie groups follow from Theorem 1.1 and Theorems 1.2, 1.3, and 1.4 below, which give the $v_{1}$-periodic homotopy groups of the factors occurring in 1.1 . We leave it to the interested reader to use these ingredients to tabulate $v_{1}^{-1} \pi_{*}(X ; p)$ for each exceptional Lie group $X$ and each prime $p$ for which $X$ is torsionfree; it is completely straightforward. We also point out that, for each such pair $(X, p)$, distinct factors in the splitting of $X_{(p)}$ given in 1.1 have nonoverlapping nonzero periodic homotopy groups, which, with $1.2,1.3$, and 1.4, implies that $v_{1}^{-1} \pi_{i}(X ; p)$ is a finite cyclic $p$-group for all even values of $i$.

We use the customary notation $q=2(p-1)$, and $\nu_{p}(-)$ denotes the exponent of $p$. If the context makes its value clear, the $p$ will be omitted as subscript of 
$\nu$ and also as an argument of $v_{1}^{-1} \pi_{*}(-)$. The first result, which is the linchpin of our entire approach, was proved in [15], using work of [19, 26].

Theorem 1.2. Let $p$ be an odd prime. Then

$$
\begin{aligned}
v_{1}^{-1} \pi_{2 i-1}\left(S^{2 n+1} ; p\right) & \approx v_{1}^{-1} \pi_{2 i}\left(S^{2 n+1} ; p\right) \\
& \approx \begin{cases}\mathrm{Z} / p^{\min (n, \nu(i-n)+1)} & \text { if } i \equiv n \bmod p-1 \\
0 & \text { otherwise. }\end{cases}
\end{aligned}
$$

The second result, Theorem 1.3 , states the $v_{1}$-periodic homotopy groups of certain sphere bundles over spheres. It will be proved in $\S 2$, where it is generalized to a wider class of sphere bundles over spheres.

Theorem 1.3. Let $p$ be an odd prime. Using the notation of Theorem 1.1, we have the following:

(1) The only nonzero $v_{1}$-periodic homotopy groups of $B(3,2 p+1)$ are

$$
\begin{aligned}
v_{1}^{-1} \pi_{2 p+q m-1}(B(3,2 p+1)) & \approx v_{1}^{-1} \pi_{2 p+q m}(B(3,2 p+1)) \\
& \approx \mathbf{Z} / p^{\min \left(p+1,1+\nu\left(m-p^{p-1}\right)\right)}
\end{aligned}
$$

(2) If $n>1$, then the only nonzero groups $v_{1}^{-1} \pi_{2 i}(B(2 n+1,2 n+q+1))$ are

$$
\begin{aligned}
v_{1}^{-1} \pi_{2 n+q m}( & B(2 n+1,2 n+q+1)) \\
& \approx \begin{cases}\mathbf{Z} / p^{\min (n, 2+\nu(m))} & \text { if } m \neq 1 \bmod p, \\
\mathbf{Z} / p^{\min (n+p-1,2+\nu(m-1))} & \text { if } m \equiv 1 \bmod p,\end{cases}
\end{aligned}
$$

and the group $v_{1}^{-1} \pi_{2 i-1}(B(2 n+1,2 n+q+1))$ has the same order as the group $v_{1}^{-1} \pi_{2 i}(B(2 n+1,2 n+q+1))$.

(3) The only nonzero 3-primary $v_{1}$-periodic homotopy groups of $B_{2}(3,11)$ are

$$
\begin{aligned}
v_{1}^{-1} \pi_{9+4 m}\left(B_{2}(3,11) ; 3\right) & \approx v_{1}^{-1} \pi_{10+4 m}\left(B_{2}(3,11) ; 3\right) \\
& \approx \mathrm{Z} / 3^{\min \left(6,1+\nu\left(m-2 \cdot 3^{4}\right)\right)}
\end{aligned}
$$

The third result, which will be proved in $\S 3$, gives the $v_{1}$-periodic homotopy groups of the five factors of torsion-free exceptional Lie groups which are built up from more than two spheres by fibering. It is quite clear how several of them could be fit under the same rubric, but we shall not bother with the extent to which this generalization can be made.

Theorem 1.4. For the following spaces $X$, the groups $v_{1}^{-1} \pi_{2 i}(X)$ are cyclic, and are nonzero in only the cases listed below. Moreover, $v_{1}^{-1} \pi_{2 i-1}(X)$ has the same order as $v_{1}^{-1} \pi_{2 i}(X)$. 


$$
\begin{aligned}
& \nu_{5}\left(\left|v_{1}^{-1} \pi_{2(4 m+5)}(B(3,11,19,27,35) ; 5)\right|\right) \\
& = \begin{cases}\min (6,4+\nu(m)) & \text { if } m \equiv 4,5 \bmod 5, \\
\min (10,4+\nu(m-1)) & \text { if } m \equiv 1 \bmod 5, \\
\min (14,4+\nu(m-2)) & \text { if } m \equiv 2 \bmod 5, \\
\min (18,4+\nu(m-3)) & \text { if } m \equiv 3 \bmod 5,\end{cases} \\
& \nu_{7}\left(\left|v_{1}^{-1} \pi_{2(6 m+7)}(B(3,15,27) ; 7)\right|\right) \\
& = \begin{cases}\min (8,2+\nu(m)) & \text { if } m \not \equiv 1 \bmod 7, \\
\min (14,2+\nu(m-1)) & \text { if } m \equiv 1 \bmod 7, \\
\nu_{7}\left(\left|v_{1}^{-1} \pi_{2(6 m+5)}(B(11,23,35) ; 7)\right|\right)\end{cases} \\
& = \begin{cases}\min (5,3+\nu(m)) & \text { if } m \neq \equiv 1,2 \bmod 7, \\
\min (11,3+\nu(m-1)) & \text { if } m \equiv 1 \bmod 7, \\
\min (17,3+\nu(m-2)) & \text { if } m \equiv 2 \bmod 7,\end{cases} \\
& \nu_{7}\left(\left|v_{1}^{-1} \pi_{2(6 m+7)}(B(3,15,27,39) ; 7)\right|\right) \\
& = \begin{cases}\min (8,3+\nu(m)) & \text { if } m \neq \equiv 1,2 \bmod 7, \\
\min (14,3+\nu(m-1)) & \text { if } m \equiv 1 \bmod 7, \\
\min (20,3+\nu(m-2)) & \text { if } m \equiv 2 \bmod 7,\end{cases} \\
& \nu_{7}\left(\left|v_{1}^{-1} \pi_{2(6 m+11)}(B(23,35,47,59) ; 7)\right|\right) \\
& = \begin{cases}\min (11,4+\nu(m)) & \text { if } m \neq \equiv 1,2,3 \bmod 7, \\
\min (17,4+\nu(m-1)) & \text { if } m \equiv 1 \bmod 7, \\
\min (23,4+\nu(m-2)) & \text { if } m \equiv 2 \bmod 7, \\
\min (29,4+\nu(m-3)) & \text { if } m \equiv 3 \bmod 7 .\end{cases}
\end{aligned}
$$

The significance of this long tabulation of results is (at least) 4-fold. First, they are a complete answer for a problem in homotopy theory. Second, they show more explicitly the form that the results for $\mathrm{SU}(n)$ of [13] will take. Third, their determination involves delicate calculations in the unstable Novikov spectral sequence (UNSS). We have tried to be quite careful in $\S \S 2$ and 3 to show the nonexpert how these calculations are performed. And fourth, they imply new results about $p$-exponents of this important class of spaces.

The $p$-exponent of a space $X$, denoted $\exp _{p}(X)$, is the largest $e$ such that $\pi_{*}(X)$ has an element of order $p^{e}$. It was proved in [11] that $\exp _{p}\left(S^{2 n+1}\right)=n$, and in particular does not depend upon $p$. If $v_{1}^{-1} \pi_{*}(X)$ has an element of order $p^{e}$, then so does $\pi_{*}(X)$, although not necessarily for the same value of *. Since the $p$-exponent of the total space of a fibration is $\leq$ the sum of the $p$-exponents of the base and the fiber, and since the $p$-exponent of a product is the maximum of the $p$-exponents of its factors, we have the following corollary of the above results.

\section{Corollary 1.5 .}

$$
\begin{gathered}
\exp _{p}\left(G_{2}\right)= \begin{cases}6 & \text { if } p=3 \text { or } 5 \\
5 & \text { if } p>5\end{cases} \\
\exp _{p}\left(F_{4}\right) \geq 11 \quad \text { if } p=5 \text { or } 7, \quad \exp _{p}\left(E_{6}\right) \geq 11 \quad \text { if } p=5 \text { or } 7
\end{gathered}
$$




$$
\begin{aligned}
& \exp _{p}\left(F_{4}\right)=\exp _{p}\left(E_{6}\right)= \begin{cases}12 & \text { if } p=11, \\
11 & \text { if } p>11,\end{cases} \\
& \exp _{p}\left(E_{7}\right) \geq\left\{\begin{array}{ll}
18 & \text { if } p=5, \\
17 & \text { if } 7 \leq p \leq 13,
\end{array} \quad \exp _{p}\left(E_{7}\right)= \begin{cases}18 & \text { if } p=17 \\
17 & \text { if } p>17\end{cases} \right. \\
& \exp _{p}\left(E_{8}\right) \geq 29 \quad \text { if } 7 \leq p \leq 23, \quad \exp _{p}\left(E_{8}\right)= \begin{cases}30 & \text { if } p=29 \\
29 & \text { if } p>29\end{cases}
\end{aligned}
$$

In particular, for each of the exceptional Lie groups, the $p$-exponent is not completely independent of $p$. We conjecture that all the inequalities in the above corollary can be replaced by equalities.

We close this section by reviewing the definition of $v_{1}^{-1} \pi_{*}(X ; p)$. As defined in [25], a space $X$ has an eventual $H$-space exponent at $p$ if, for some $e$ and $t, p^{e}: \Omega^{t} X \rightarrow \Omega^{t} X$ is null-homotopic. This is true whenever $X$ is a compact Lie group, because it was shown in [17] that for some $m$ the map $p^{m}: X \rightarrow X$ factors through a finite product of odd-dimensional spheres, and it was shown in [11] that odd-dimensional spheres have eventual $H$-space exponents at all primes.

Assume that $p^{e}: \Omega^{t} X \rightarrow \Omega^{t} X$ is null-homotopic, and let $M^{k}$ denote a mod- $p^{e}$ Moore space with top cell of dimension $k$. Let $q=2(p-1)$, let $M^{k+q p^{e}} \stackrel{A}{\rightarrow} M^{k}$ denote an Adams map [1], and let $\operatorname{Map}_{*}(-,-)$ be the space of pointed maps. The null homotopy defines a map $s$, which is used in the definition of the composite below, denoted $A^{\prime}$.

$$
\Omega^{t} X \stackrel{s}{\rightarrow} \operatorname{Map}_{*}\left(M^{t+1}, X\right) \stackrel{A^{*}}{\rightarrow} \operatorname{Map}_{*}\left(M^{t+q p^{e}+1}, X\right) \stackrel{j^{*}}{\rightarrow} \Omega^{t+q p^{e}} X .
$$

The $v_{1}$-periodic homotopy groups of $X$ are defined by applying $\pi_{*}(-)$ to (1.6), iterating, and passing to the direct limit using the maps $A^{\prime}$.

$$
v_{1}^{-1} \pi_{n}(X)=\operatorname{dir} \lim \pi_{n-t}\left(\Omega^{t+i q p^{e}}(X)\right) .
$$

This definition, originally given in [13], demanded some care in choosing $e$, $s$, and $A$ in order that the definition be compatible with fibrations and with periodic mod- $p^{e}$ homotopy groups. Later, in [16], the definition was generalized in a way which applies to all spaces and avoids all choices. Note that the definition as a direct limit implies that if $v_{1}^{-1} \pi_{n}(X)$ is finitely generated it will be a direct summand of $\pi_{n+i q p^{e}}(X)$ for $i$ sufficiently large. We make no attempt to estimate how small such $i$ can be chosen, i.e., in what actual homotopy groups the periodic groups exist as direct summands. The first two authors hope to address this in a subsequent paper.

\section{2. $v_{1}$-PERIODIC HOMOTOPY GROUPS OF SPHERE BUNDLES OVER SPHERES}

In this section we prove two results which give the precise $v_{1}$-periodic homotopy groups of certain sphere bundles over spheres. One of these is Theorem 1.3(2), while the other is the following result, which implies parts (1) and (3) of Theorem 1.3. As throughout this paper, $p$ denotes an odd prime, and $q=2(p-1)$.

Theorem 2.1. Let $S^{3} \rightarrow B_{k}(3, k q+3) \rightarrow S^{k q+3}$ be a fibration with attaching map $\alpha_{k}$ for the bottom cells of $B_{k}(3, k q+3)$. Then the only nonzero $v_{1}$-periodic 
homotopy groups of $B_{k}(3, k q+3)$ are

$$
\begin{aligned}
v_{1}^{-1} \pi_{q(k+m)+1}\left(B_{k}(3, k q+3)\right) & \approx v_{1}^{-1} \pi_{q(k+m)+2}\left(B_{k}(3, k q+3)\right) \\
& \approx \mathbf{Z} / p^{\min \left(k(p-1)+2,1+\nu\left(m-k p^{k(p-1)}\right)\right)} .
\end{aligned}
$$

The proof of both Theorems 1.3 and 2.1 begins by reducing to a calculation of the 1-line of the UNSS. This reduction is established as in [13] by building the space up by fibrations of spheres and studying the morphism from the exact sequence of $v_{1}^{-1} \pi_{*}(-)$ to that of $E_{2}^{*, *}(-)$. For spheres, this morphism is a bijection from even groups to the 1-line and an injection from odd groups to the 2-line, and this implies that the same is true for the built-up spaces. The morphism is only defined in large dimensions, but that suffices for all since both are periodic. This periodicity was proved for $E_{2}^{1, *}(\mathrm{SU}(n))$ in [13] using results of $[3,12]$. For the factors of exceptional Lie groups which are not also factors of some $\mathrm{SU}(n)$, this periodicity will be a corollary of the calculation performed here. This exact-sequence argument was used in [5] to prove a result which generalizes that which is needed here.

We shall not attempt to calculate $E_{2}^{2, *}(-)$ for the spaces involved in Theorems 1.3 and 2.1, for the 2-line seems to be more complicated, being apparently not always cyclic. Instead, we note, as was done in [13], that the fact that $v_{1}^{-1} \pi_{2 i-1}\left(S^{2 n+1}\right)$ and $v_{1}^{-1} \pi_{2 i}\left(S^{2 n+1}\right)$ have the same order allows us to deduce the same thing for spaces built up from fibrations of spheres, using a simple Euler characteristic argument. A special argument will be used in Theorem 2.24 to show that the odd $v_{1}$-periodic homotopy groups of the spaces in Theorem 2.1 are cyclic.

Remark. Theorems 1.3 and 2.1 can be interpreted as giving explicit formulas for the numbers called $e_{p}(k, n)$ in [13], $d_{p}^{K}(k+1, n)$ in [12], and $\nu_{p}(\Delta(k, n))$ in [18] for certain values of $k$ and $n$. Here we have a result in number theory proved via the UNSS. For example, the second author conjectured the following result based on computer calculations and was unable to prove it using methods of number theory, but it follows immediately from Theorem 1.3(1) and [13, 1.4]. Recall that the Stirling numbers of the second kind, denoted by $S(k, j)$, are defined by

$$
\left(e^{x}-1\right)^{j}=\sum j ! S(k, j) \frac{x^{k}}{k !} .
$$

Proposition 2.2. Let $e_{p}(k, n)=\min \left\{\nu_{p}(j ! S(k, j)): n \leq j \leq k\right\}$, where $S(k, j)$ denotes a Stirling number of the second kind. If $p$ is odd, then for any $m>0$

$$
e_{p}((p-1)(m+1)+1, p+1)=\min \left(p+1,1+\nu_{p}\left(m-p^{p-1}\right)\right) .
$$

We follow quite closely the proof of $[2,4.11]$, which was essentially our Theorem 1.3(1), i.e., the case $k=1$ of Theorem 2.1. We point out that our current work brought to light a minor mistake in $[2,4.11]$. The $(-1)^{s+1}$ should be replaced by 1 .

It was shown in [7] that the only nonzero groups $E_{2}^{1, *}\left(S^{2 n+1}\right)$ are

$$
E_{2}^{1,2 n+q m+1}\left(S^{2 n+1}\right) \approx \mathbf{Z} / p^{\min (n, \nu(m)+1)} .
$$

The orders of the groups in Theorems 2.1 and 1.3(2) follow from the above remarks and the following results. 
Theorem 2.4. Assume $n>1$. In the exact sequence

$$
\begin{aligned}
0 & \rightarrow E_{2}^{1,2 n+q m+1}\left(S^{2 n+1}\right) \stackrel{i_{*}}{\rightarrow} E_{2}^{1,2 n+q m+1}(B(2 n+1,2 n+q+1)) \\
& \stackrel{j_{*}}{\rightarrow} E_{2}^{1,2 n+q m+1}\left(S^{2 n+q+1}\right) \stackrel{\partial}{\rightarrow} E_{2}^{2,2 n+q m+1}\left(S^{2 n+1}\right)
\end{aligned}
$$

the morphism $\partial$ is 0 unless

- $n+p-2 \leq \nu(m-1)$, when it is a surjection $\mathbf{Z} / p^{n+p-1} \rightarrow \mathbf{Z} / p$, or

- $n-2<\nu(m)$, when it is an injection $\mathbf{Z} / p \rightarrow \mathbf{Z} / p^{n}$.

Theorem 2.5. In the exact sequence

$$
\begin{aligned}
& 0 \rightarrow E_{2}^{1, q(k+m)+3}\left(S^{3}\right) \stackrel{i_{*}}{\rightarrow} E_{2}^{1, q(k+m)+3}\left(B_{k}(3, k q+3)\right) \\
& \quad \stackrel{j_{*}}{\rightarrow} E_{2}^{1, q(k+m)+3}\left(S^{k q+3}\right) \stackrel{\partial}{\rightarrow} E_{2}^{2, k q+3}\left(S^{3}\right)
\end{aligned}
$$

the morphism $\partial$ is a surjection to $\mathbf{Z} / p$ unless

$$
\nu(m) \geq k(p-1) \text { and } k \equiv m / p^{k(p-1)} \bmod p,
$$

in which case it is 0 .

After proving these theorems, we will prove in Theorem 2.23 that the groups $E_{2}^{1,2 n+q m+1}(B(2 n+1,2 n+q+1))$ and $E_{2}^{1, q(k+m)+3}\left(B_{k}(3, k q+3)\right)$ are cyclic, which, with the earlier remarks and Theorem 2.24 for the cyclicity of some $v_{1}^{-1} \pi_{2 i-1}(-)$, completes the proof of Theorems 1.3(2) and 2.1.

We now embark upon the proof of Theorems 2.4 and 2.5, which involves careful UNSS calculations similar to those of [2]. We will review the relevant background results as we proceed. We use the Hazewinkel generators $v_{i} \in B P_{*}$ and $h_{i}=c\left(t_{i}\right) \in B P_{*}(B P)$. We abbreviate $\eta_{R}(-)$ to $\eta(-)$, and write $h_{i} v_{j}$ interchangeably with $\eta\left(v_{j}\right) h_{i}$. We will need the following result, whose proof we outline, although it involves standard $B P$ methods, such as those of [24].

Lemma 2.6. (i) $\psi\left(h_{2}\right)=h_{2} \otimes 1+1 \otimes h_{2}+\sum_{i=1}^{p-1} \frac{1}{p}\left(\begin{array}{l}p \\ i\end{array}\right) h_{1}^{i} \otimes h_{1}^{p-i} v_{1}+h_{1}^{p} \otimes h_{1}$;

(ii) $v_{1}=p h_{1}+\eta\left(v_{1}\right)$;

(iii) $v_{2}=p h_{2}+\left(1-p^{p-1}\right) h_{1}^{p} v_{1}+\eta\left(v_{2}\right)-(p+1) v_{1}^{p} h_{1}+\sum_{i=2}^{p} a_{i} v_{1}^{p+1-i} p^{i} h_{1}^{i}$, where $a_{i} \in \mathbf{Z}$.

Proof. We use $v_{1}=p m_{1}$ and $p v_{2}+v_{1}^{p+1}=p^{2} m_{2}$. Part (i) follows from

$$
\sum_{i, l} \psi\left(h_{i}\right)^{p^{l}} m_{l}=\sum_{j, k, l} h_{k}^{p^{j+l}} \otimes h_{j}^{p^{l}} m_{l} .
$$

Part (ii) is obtained by multiplying $m_{1}=h_{1}+\eta\left(m_{1}\right)$ by $p$. Part (iii) is obtained by multiplying $m_{2}=h_{2}+\eta\left(m_{1}\right) h_{1}^{p}+\eta\left(m_{2}\right)$ by $p^{2}$. This yields

$$
p v_{2}+v_{1}^{p+1}=p^{2} h_{2}+p h_{1}^{p} v_{1}+p \eta\left(v_{2}\right)+\left(v_{1}-p h_{1}\right)^{p+1}
$$

In the expansion of the last term, the $v_{1}^{p+1}$ cancels with the term on the left-hand side, after which we divide everything by $p$. The next term in the expansion appears in our stated formula, while the last two terms combine to $-v_{1} p^{p} h_{1}^{p}-$ $p^{p-1} h_{1}^{p} v_{1}$. The first of these appears in the sum in our formula, and the second is combined with an earlier term. 
The UNSS is defined so that $E_{2}^{s, t}\left(S^{2 n+1}\right)$ is the homology of the unstable cobar complex, which has, for example, terms with $s=2$ spanned by those $v^{a} h^{b} \otimes v^{c} h^{d} \otimes v^{e} l_{2 n+1}$ satisfying

$$
|d| \leq \frac{1}{2}\left|v^{e}\right|+n \text { and }|b| \leq \frac{1}{2}\left|v^{c}\right|+\frac{1}{2}\left|h^{d}\right|+\frac{1}{2}\left|v^{e}\right|+n .
$$

Here monomials such as $h^{b}$ are $h_{1}^{b_{1}} h_{2}^{b_{2}} \ldots$ with $|b|=b_{1}+b_{2}+\cdots$ and

$$
\frac{1}{2}\left|h^{b}\right|=(p-1) b_{1}+\left(p^{2}-1\right) b_{2}+\cdots .
$$

The factor $v^{c}$ can be considered as acting on the right of $h^{b}$ or on the left of $h^{d}$. Terms such as these are sometimes written in [ | | ] notation. The boundary $d$ in the unstable cobar complex is defined as in [7, p. 244]; for example,

$$
\begin{aligned}
d\left(v^{a} h^{b} v^{c} l_{2 n+1}\right)= & \left(\eta\left(v^{a}\right)-v^{a}\right) \otimes h^{b} v^{c} l_{2 n+1}-v^{a} \bar{\psi}\left(h^{b}\right) v^{c} l_{2 n+1} \\
& -v^{a} h^{b} \otimes\left(\eta\left(v^{c}\right)-v^{c}\right) l_{2 n+1},
\end{aligned}
$$

where $\bar{\psi}\left(h^{b}\right)=\psi\left(h^{b}\right)-h^{b} \otimes 1-1 \otimes h^{b}$. For spaces such as those of Theorem 2.5, $E_{2}^{s, t}(B(2 n+1,2 n+q+1))$ has terms ending in $x_{2 n+1}$ or $x_{2 n+q+1}$, and $d\left(v^{a} h^{b} v^{c} x_{2 n+q+1}\right)$ has the additional term $v^{a} h^{b} \otimes v^{c} h_{1} x_{2 n+1}$. In $\S 3$, we will consider more explicitly how the coaction in $B P_{*}(\mathrm{SU}(n))$ comes into play in the boundary in the cobar complex of $\mathrm{SU}(n)$ and its quotients.

We expand upon (2.3) by recalling the following result from [7, 9.12].

Proposition 2.9. Let $\mu=\min (n, \nu(m)+1)$. Then

$$
E_{2}^{1,2 n+q m+1}\left(S^{2 n+1}\right) \approx \mathbf{Z} / p^{\mu}
$$

with generator $\alpha_{m / \mu}=d\left(v_{1}^{m}\right) / p^{\mu}$ satisfying

$$
\text { if } \mu=n \text {, then } \alpha_{m / n} \equiv-v_{1}^{m-n} h_{1}^{n} \bmod E_{1}\left(S^{2 n-1}\right) \text {. }
$$

The double suspension homomorphism

$$
E_{2}^{1,2 n+q m-1}\left(S^{2 n-1}\right) \stackrel{\Sigma^{2}}{\rightarrow} E_{2}^{1,2 n+q m+1}\left(S^{2 n+1}\right)
$$

is injective.

The abbreviation $\alpha_{m}$ will be used for $\alpha_{m / 1}$. Note that $\alpha_{1}=-h_{1}$, which will be used frequently without comment. Although there should be no confusion between these classes and homotopy classes of the same name such as those mentioned in 1.1, the two are compatible anyway; the $E_{2}$-classes $\alpha_{m}$ detect the homotopy classes $\alpha_{m}$. A variation on (2.7) which will frequently be used is that for $\alpha_{m / j} \otimes h^{d} v^{e} l_{2 n+1}$ to be defined, the second condition of (2.7) should be replaced by

$$
j \leq \frac{1}{2}\left|h^{d}\right|+\frac{1}{2}\left|v^{e}\right|+n .
$$

Next we review results from [2] about the unstable elements on the 2-line. The double suspension Hopf invariant $H_{2}$ is discussed there. We denote by $H^{\prime}$ the morphism

$$
H^{\prime}: E_{2}^{2}\left(S^{2 n+1}\right) \stackrel{H_{2}}{\rightarrow} \operatorname{Ext}_{\mathscr{C}}^{1}(W(n)) \rightarrow E_{2}^{1}(M)
$$

obtained by following $\mathrm{H}_{2}$ by the stabilization. Here

$$
E_{2}^{s}(M) \approx \operatorname{Ext}_{B P_{*} B P}^{S}\left(B P_{*}, B P_{*} / p\right)
$$

denotes the $E_{2}$-term of the stable UNSS for the $\bmod p$ Moore spectrum $M$. We will eventually need the following facts about $E_{2}(M)$. 
Lemma 2.11. (1) $E_{2}(M)$ is commutative.

(2) $v_{1}^{k} h_{1} \neq 0 \in E_{2}^{1}(M)$.

(3) If $x \in E_{2}(M)$, then $v_{2} x=x v_{2}=0$.

(4) $v_{1} h_{1}^{k(p-1)+1}=v_{1}^{k(p-1)+1} h_{1}$ in $E_{2}(M)$.

(5) If $s \not \equiv 0 \bmod p$, then $\alpha_{s p^{e-1} / e}=-s v_{1}^{s p^{e-1}-1} h_{1}$ in $E_{2}(M)$.

Proof. Part (1) is well known, part (2) is [24, p. 157], and part (3) follows from $[20,2.10]$. Part (4) follows from Proposition 2.6(iii), part 3 of this lemma, and the fact that $p h_{1}=0 \in E_{2}(M)$. To prove part (5), we use 2.6(ii) to expand $v_{1}^{s p^{e-1}}$, obtaining

$$
\alpha_{s p^{e-1} / e}=\frac{1}{p^{e}}\left(\eta\left(v_{1}^{s p^{e-1}}\right)-\left(p h_{1}+\eta\left(v_{1}\right)\right)^{s p^{e-1}}\right)=-\sum_{j=1}^{s p^{e-1}}\left(\begin{array}{c}
s p^{e-1} \\
j
\end{array}\right) p^{j-e} h_{1}^{j} v_{1}^{s p^{e-1}-j}
$$

All terms except $j=1$ are divisible by $p$, and hence 0 . To insure that terms with $j$ large are $p$ times an admissible element, write $p^{j-e} h_{1}^{j}$ as $p\left(v_{1}-\eta\left(v_{1}\right)\right)^{j-e-1} h^{e+1}$.

Now we cull from [2] the following result. We denote by $\widetilde{E}_{2}^{2, t}\left(S^{2 n+1}\right)$ the subgroup of $E_{2}^{2, t}\left(S^{2 n+1}\right)$ consisting of unstable elements, i. e., those which stabilize to zero.

Theorem 2.12. (1) Let a be fixed, and $t=\nu(a)$. The groups $\widetilde{E}_{2}^{2, q a+2 n+1}\left(S^{2 n+1}\right)$ are

(a) $\mathbf{Z} / p^{n}$ if $n \leq t+1$, injecting to one another under $\Sigma^{2}$;

(b) $\mathbf{Z} / p^{t+1}$ if $t+1 \leq n<a-t$, with $\Sigma^{2}$ inducing $\cdot p$;

(c) $\mathbf{Z} / p^{a-n}$ if $a-t-1 \leq n<a$, surjecting to one another under $\Sigma^{2}$.

(2) Suppose $x \in \widetilde{E}_{2}^{2}\left(S^{2 n+1}\right)$ may be represented in the unstable cobar complex by a cycle of the form $\gamma \otimes h_{1}^{n} l_{2 n+1} \bmod E_{1}\left(S^{2 n-1}\right)$, where $\gamma_{2 p n-1}$ is defined. (Note that this $2 p n-1$ is 2 smaller than necessary for $\gamma \otimes h_{1}^{n} l_{2 n+1}$ to be defined.) Then

$$
H^{\prime}(x)=\gamma \in E_{2}^{1}(M),
$$

and $x$ double desuspends if and only if $\gamma=0$.

(3) In $E_{2}^{2, q a+2 n+1}\left(S^{2 n+1}\right)$ with $t=\nu(a)$

(a) $\alpha_{a-1} \otimes \alpha_{1} l_{2 n+1}$ has order $p$ if $1 \leq n \leq t+1$, while if $n>t+1$, it is 0 and equals $d\left(v_{1}^{a-t-2} h_{1}^{t+2} l_{2 n+1}\right)$ in the cobar complex;

(b) $\alpha_{a-2} \otimes h_{1}^{2} l_{2 n+1}$ has order $p^{2}$ if $2 \leq n \leq t+1$, has order $p$ if $n=t+2$, and is $d\left(v_{1}^{a-t-3} h_{1}^{t+3} l_{2 n+1}\right)$ if $n \geq t+3$;

(c) $\alpha_{a-3} \otimes h_{1}^{3} l_{7}$ is a generator when $n=3$.

Proof. Part (1) is [2, 3.2], and the formula for $H^{\prime}(x)$ is part of [4, 5.3]. The double suspension EHP sequence implies that if $x$ double desuspends, then $H^{\prime}(x)=0$. If $x$ does not double desuspend, then [2, 3.2(iv)] implies that $H^{\prime}(x) \neq 0$. In part 3 , the nonzero parts are most easily seen in the tables on p. 534 and 530 of [2]. The part which represents them as a boundary when they become 0 is from $[2,3.7]$. The formulas there suspend to the desired boundaries

Now we can prove Theorem 2.4. We begin with the case $\nu(m-1)=0$, in which case $\partial: \mathbf{Z} / p \rightarrow \mathbf{Z} / p^{\min (\nu(m)+1, n)}$. Similarly to [8] or [2, 4.5], the image of 
a generator is $\alpha_{m-1} \alpha_{1} l_{2 n+1}$. By (2.10), $\alpha_{m-1} \alpha_{1} l_{2 n+1}$ desuspends to $S^{3}$, where it is nonzero. To see that $\alpha_{m-1} \alpha_{1} l_{3} \neq 0$, we use Proposition 2.9 to see that $\bmod E_{2}^{1}\left(S^{1}\right)$ it is $v_{1}^{m-2} h_{1} \otimes h_{1} l_{3}$, which satisfies

$$
H^{\prime}\left(v_{1}^{m-2} h_{1} \otimes h_{1} l_{3}\right)=v_{1}^{m-2} h_{1} \neq 0,
$$

by Theorem 2.12(2) and Lemma 2.11. Alternatively, just apply 2.12(3).

By Theorem 2.12(1),

$$
\widetilde{E}_{2}^{2, q m+3}\left(S^{3}\right) \rightarrow \widetilde{E}_{2}^{2, q m+2 n+1}\left(S^{2 n+1}\right)
$$

is nonzero if and only if $n \leq \nu(m)+1$. Hence $\alpha_{m-1} \alpha_{1} l_{2 n+1} \neq 0$ if and only if $n \leq \nu(m)+1$, as desired.

Now assume $\nu(m-1)>0$. Then $\widetilde{E}_{2}^{2,2 n+q m+1}\left(S^{2 n+1}\right) \approx \mathbf{Z} / p$, and so by Theorem 2.12(1)

$$
\partial=0 \quad \text { if and only if } \partial \text { (gen) double desuspends. }
$$

We divide into two cases, the first being $\nu(m-1)<n+p-2$. Letting $e=$ $\nu(m-1)+1$,

$$
\partial(\text { gen })=\alpha_{m-1 / e} \alpha_{1} l_{2 n+1} .
$$

By Proposition 2.9, $\alpha_{m-1 / e}$ desuspends to $S^{2 e+1}$. The assumption implies

$$
2 e+1 \leq q+2 n-1
$$

so that $\alpha_{m-1 / e} \alpha_{1} l_{2 n+1}$ double desuspends, hence is 0 , as claimed.

The final case is $\nu(m-1) \geq n+p-2$. In this case $\partial($ gen $)=\alpha_{m-1 / n+p-1} \alpha_{1} l_{2 n+1}$, which, by Proposition 2.9, equals $v_{1}^{K} h_{1}^{n+p-1} \otimes h_{1} l_{2 n+1}$, mod terms which desuspend, where $K=m-1-n-p+1$. By Lemma 2.13 below, in the unstable cobar complex

$$
h_{1}^{p} \otimes h_{1}^{n} l_{2 n+1}+h_{1}^{n+p-1} \otimes h_{1} l_{2 n+1}
$$

is a boundary mod terms that desuspend. Hence

$$
H^{\prime}\left(v_{1}^{K} h_{1}^{n+p-1} \otimes h_{1} l_{2 n+1}\right)=-H^{\prime}\left(v_{1}^{K} h_{1}^{p} \otimes h_{1}^{n} l_{2 n+1}\right)=-v_{1}^{K} h_{1}^{p}=-v_{1}^{K+p-1} h_{1} \neq 0,
$$

where the first " = " follows from 2.12(2), the last " = " follows from 2.11(4), and the " $\neq$ " follows from 2.11(2). Hence by 2.12(2), $\partial \neq 0$, as claimed.

The following lemma was used in the above proof, and will be used again later.

Lemma 2.13. For $n \geq 1$ and $K \geq 0$,

$$
\left(h_{1}^{p} \otimes h_{1}^{K(p-1)+n}+h_{1}^{K(p-1)+p+n-1} \otimes h_{1}\right) v_{1}^{K} l_{2 n+1}
$$

is the sum of a boundary and a class which double desuspends.

Letting $n=1$ and $K=0$, and noting that 2 is a unit, we obtain the following corollary.

Corollary 2.14. $h_{1}^{p} \otimes h_{1} l_{3}$ is the sum of a boundary and a class which desuspends to $S^{1}$.

Proof of Lemma 2.13. The class which bounds the stated class mod elements which double desuspend is $h_{1}^{e} h_{2} v_{1}^{K} l_{2 n+1}$, where $e=K(p-1)+n-1$. One term in the boundary of this is

$$
h_{1}^{e} h_{2} \otimes d\left(v_{1}^{K}\right) l_{2 n+1}=-h_{1}^{e} h_{2} \otimes p \alpha_{K} l_{2 n+1},
$$


which double desuspends. The other part of the boundary is

$$
\begin{aligned}
\sum_{i=0}^{e}\left(\begin{array}{l}
e \\
i
\end{array}\right)\left(h_{1}^{i} \otimes h_{1}^{e-i} h_{2}+h_{1}^{i} h_{2} \otimes\right. & h_{1}^{e-i}+h_{1}^{i+p} \otimes h_{1}^{e-i+1} \\
& \left.+\sum_{j=1}^{p-1} a_{j} h_{1}^{i+j} \otimes h_{1}^{e-i+p-j} v_{1}\right) v_{1}^{K} l_{2 n+1},
\end{aligned}
$$

with the terms $\left(1 \otimes h_{1}^{e} h_{2}+h_{1}^{e} h_{2} \otimes 1\right) v_{1}^{K} l_{2 n+1}$ omitted. All terms double desuspend except the cases $i=0$ and $i=e$ of the third term in the sum, yielding the claim.

Another corollary which we will use several times in $\S 3$ is

Corollary 2.15. For any $e \geq 0, v_{1}^{e} h_{1}^{p-1+n} \otimes h_{1} l_{2 n+1} \neq 0 \in E_{2}^{2, *}\left(S^{2 n+1}\right)$.

Proof. By Lemma 2.13, we can rewrite it so that its Hopf invariant can be calculated by $2.12(2)$ to be $v_{1}^{e} h_{1}^{p}$, which 2.11 shows to be nonzero.

Now we begin the proof of Theorem 2.5. We begin with the case $\nu(m)<$ $k(p-1)$. In this case,

$$
\partial(\text { gen })=\alpha_{m / \nu(m)+1} \alpha_{k} l_{3} \equiv-\alpha_{m / \nu(m)+1} v_{1}^{k-1} h_{1} l_{3},
$$

mod terms that desuspend to $S^{1}$. Here we have used 2.9. The assumption that $\nu(m)<k(p-1)$ implies $\alpha_{m / \nu(m)+1}$ is defined on $S^{k q+1}$, and hence 2.12(2) implies

$$
H^{\prime}(\partial(\text { gen }))=-\alpha_{m / \nu(m)+1} v_{1}^{k-1} \neq 0,
$$

where the last step uses parts (1), (5), and (2) of Lemma 2.11. Thus $\partial \neq 0$ in this case, as claimed.

Now we complete the proof of 2.5 by considering the case $\nu(m) \geq k(p-1)$. We let $r=k(p-1)+1, s=m / p^{\nu(m)}$, and

$$
\varepsilon= \begin{cases}0 & \text { if } \nu(m)>k(p-1), \\ 1 & \text { if } \nu(m)=k(p-1) .\end{cases}
$$

We will establish the following string of equations in the next paragraph, and then we will further analyze whether these terms are 0 by studying their Hopf invariants. The following string is valid mod terms which desuspend to $S^{1}$ :

$$
\begin{aligned}
\partial(\text { gen }) & =\alpha_{m / r} \alpha_{k} l_{3} \\
& =p^{-r}\left(\eta\left(v_{1}^{m}\right)-\left(p h_{1}+\eta\left(v_{1}\right)\right)^{m}\right) \alpha_{k} l_{3} \\
& =-\sum_{j=1}^{m}\left(\begin{array}{c}
m \\
j
\end{array}\right) p^{j-r} h_{1}^{j} v_{1}^{m-j} \alpha_{k} l_{3} \\
& =-p^{m-r} h_{1}^{m} \alpha_{k} l_{3}-\varepsilon s h_{1} v_{1}^{m-1} \alpha_{k} l_{3} \\
& =-v_{1}^{m-r} h_{1}^{r} \alpha_{k} l_{3}-v_{1}^{m-r-1} h_{1}^{r} v_{1}^{k} h_{1} l_{3}+\varepsilon s h_{1} v_{1}^{m+k-2} h_{1} l_{3} \\
& =A+B+C,
\end{aligned}
$$

where $A, B$, and $C$ denote the three terms in the preceding line.

Line (2.17) follows from 2.9, (2.8), and 2.6(ii). Line (2.18) has been obtained by observing that in the sum all terms desuspend to $S^{1}$ except $j=m$ and, if 
$\nu(m)=r-1$, then $j=1$. To see this, we observe that we need to have a $p$ to make $\alpha_{k} l_{3}$ desuspend. This will be true unless $j=1$ and $\nu(m)=r-1$. The requirement that $j$ be less than half the degree of the symbols following $h_{1}^{j}$ will only be a problem for large values of $j$. When $j$ is large, write the term as

$$
\left(\begin{array}{c}
m \\
j
\end{array}\right) p\left(v_{1}-\eta\left(v_{1}\right)\right)^{j-r-1} h_{1}^{r+1} v_{1}^{m-j} \alpha_{k} l_{3} .
$$

Since $p$ times anything which is defined on $S^{3}$ desuspends to $S^{1}$, this desuspends to $S^{1}$ provided

$$
k(p-1)+2=r+1 \leq(p-1)(m-j+k)+1, \quad 1 \leq(p-1)(m-j), j<m .
$$

To obtain (2.19), we have rewritten the first term of (2.18) as

$$
-p\left(v_{1}-\eta\left(v_{1}\right)\right)^{m-r-1} h_{1}^{r+1} \alpha_{k} l_{3},
$$

and observed that when this is expanded, all terms except the first desuspend; we write $p h_{1}=v_{1}-\eta\left(v_{1}\right)$ in that first term. We have also converted $\alpha_{k}$ to $-v_{1}^{k-1} h_{1}$ at several places, using 2.9.

We note that we can evaluate the Hopf invariant of $B$ and $C$ by 2.12(2), and, using 2.11 and recalling $r=k(p-1)+1$, we obtain

$$
H^{\prime}(B+C)=(-1+\varepsilon s) v_{1}^{m+k-2} h_{1} .
$$

We will show that $H^{\prime}(A)=(1-k) v_{1}^{m+k-2} h_{1}$, which will complete the proof of Theorem 2.5. If $k=1$, then $A=v_{1}^{m-r+k-1} h_{1}^{p} \otimes h_{1} l_{3}$, which by Corollary 2.14 is a boundary mod $S^{1}$, and hence its Hopf invariant is 0 , as claimed.

Now assume $k>1$, and define $A^{\prime}$ by $A=v_{1}^{m-r} A^{\prime}$. Then

$$
\begin{aligned}
A^{\prime} & =-h_{1}^{r} \alpha_{k} l_{3}=-h_{1}^{r} \frac{1}{p}\left(\eta\left(v_{1}^{k}\right)-\left(p h_{1}+\eta\left(v_{1}\right)\right)^{k}\right) l_{3} \\
& =\sum_{j=1}^{k}\left(\begin{array}{c}
k \\
j
\end{array}\right) h_{1}^{r} \otimes p^{j-1} h_{1}^{j} v_{1}^{k-j} l_{3} \\
& =k h_{1}^{r} \otimes h_{1} v_{1}^{k-1} l_{3}+\sum_{j=2}^{k}\left(\begin{array}{l}
k \\
j
\end{array}\right) h_{1}^{r} p\left(v_{1}-\eta\left(v_{1}\right)\right)^{j-2} h_{1}^{2} v_{1}^{k-j} l_{3} .
\end{aligned}
$$

Thanks to the extra $p$, the terms in the sum with $j<k$ desuspend to $S^{1}$, but the $j=k$ term has a problem because of the $h_{1}^{2} l_{3}$ at the end. When its $\left(v_{1}-\eta\left(v_{1}\right)\right)^{k-2}$ is expanded, all terms except the first will desuspend because $\eta\left(v_{1}\right) h_{1}^{2}=h_{1}^{2} v_{1}$. Thus, always working $\bmod S^{1}$, we obtain

$$
\begin{aligned}
A^{\prime} & =k h_{1}^{r} \otimes h_{1} v_{1}^{k-1} l_{3}+h_{1}^{r} p v_{1}^{k-2} h_{1}^{2} l_{3} \\
& =k h_{1}^{k(p-1)+1} \otimes h_{1} v_{1}^{k-1} l_{3}+h_{1}^{k(p-1)+1} v_{1}^{k-1} h_{1} l_{3}-h_{1}^{k(p-1)+1} v_{1}^{k-2} h_{1} v_{1} l_{3},
\end{aligned}
$$

where we have used 2.6(ii) in the last line.

We analyze the terms of (2.21) one at a time. By Lemma 2.22(b) and Corollary 2.14 , the second term yields

$$
h_{1}^{k(p-1)+1} v_{1}^{k-1} h_{1} l_{3}=v_{1}^{(k-1) p} h_{1}^{p} \otimes h_{1} l_{3} \sim 0 .
$$


For the third term, we have, mod boundaries and classes that desuspend,

$$
\begin{aligned}
-h_{1}^{k(p-1)+1} v_{1}^{k-2} h_{1} v_{1} l_{3} & =-v_{1}^{p(k-2)} h_{1}^{2 p-1} \otimes h_{1} v_{1} l_{3}=v_{1}^{p(k-2)} h_{1}^{p} \otimes h_{1}^{p} v_{1} l_{3} \\
& =v_{1}^{p(k-2)} h_{1}^{p} v_{1}^{p} h_{1} l_{3},
\end{aligned}
$$

where we have used Lemma 2.22(c), then Lemma 2.13, and then Lemma 2.22(a). For the first term of (2.21), we have

$$
k h_{1}^{k(p-1)+1} \otimes h_{1} v_{1}^{k-1} l_{3} \equiv-k h_{1}^{p} \otimes h_{1}^{(k-1)(p-1)+1} v_{1}^{k-1} l_{3}=-k h_{1}^{p} v_{1}^{(k-1) p} h_{1} l_{3},
$$

by Lemma 2.13 followed by Lemma $2.22(a)$. Substituting these into (2.21), we can apply 2.12(2) to find

$$
H^{\prime}(A)=(1-k) v_{1}^{m-r} v_{1}^{p(k-1)} h_{1}^{p},
$$

which by 2.11 equals $(1-k) v_{1}^{m+k-2} h_{1}$, as desired.

The following lemma was used several times in the preceding paragraph.

Lemma 2.22. In the cobar complex of $S^{3}$, mod terms that desuspend to $S^{1}$,

(a) $h_{1}^{(k-1)(p-1)+1} v_{1}^{k-1} l_{3} \equiv v_{1}^{(k-1) p} h_{1} l_{3}$;

(b) $h_{1}^{k(p-1)+1} v_{1}^{k-1} h_{1} l_{3} \equiv v_{1}^{(k-1) p} h_{1}^{p} \otimes h_{1} l_{3}$;

(c) $h_{1}^{k(p-1)+1} v_{1}^{k-2} h_{1} v_{1} l_{3} \equiv v_{1}^{p(k-2)} h_{1}^{2 p-1} \otimes h_{1} v_{1} l_{3}$.

Proof. We multiply Lemma 2.6(iii) by

(a) $\quad\left(h_{1}^{(k-2)(p-1)} v_{1}^{k-2}+v_{1}^{p} h_{1}^{(k-3)(p-1)} v_{1}^{k-3}+\cdots+v_{1}^{(k-2) p} \cdot 1\right) \cdot l_{3}$;

(b) $\left(h_{1}^{(k-1)(p-1)} v_{1}^{k-2}+v_{1}^{p} h_{1}^{(k-2)(p-1)} v_{1}^{k-3}+\cdots+v_{1}^{(k-2) p} h_{1}^{p-1}\right) \otimes h_{1} l_{3}$;

(c) $\left(h_{1}^{(k-1)(p-1)} v_{1}^{k-3}+v_{1}^{p} h_{1}^{(k-2)(p-1)} v_{1}^{k-4}+\cdots+v_{1}^{(k-3) p} h_{1}^{2(p-1)}\right) \otimes h_{1} v_{1} l_{3}$.

Clearly the indicated sum, when multiplied by the $h_{1}^{p} v_{1}-v_{1}^{p} h_{1}$ which is part of 2.6(iii), yields the desired result, with intermediate terms cancelling. We must show that when the indicated sum is multiplied by the other terms of 2.6(iii), all terms obtained desuspend to $S^{1}$. In parts (a) and (c), the terms in each sum already desuspend, and this cannot be hurt by multiplying by the other terms of 2.6(iii). Part (b) is slightly more difficult because the terms of the sum do not desuspend to $S^{1}$ because $h_{1} l_{3}$ requires a $p$ in order to desuspend. When terms of the sum are multiplied by $v_{2}, \eta\left(v_{2}\right), p h_{2}, p^{p-1} h_{1}^{p} v_{1}$, or $p^{i} h_{1}^{i}$, the Hopf invariant may be evaluated using 2.12(2). In the first two cases, this yields 0 by 2.11(3). In the other cases, we use the fact that if $x \in \operatorname{cobar}\left(S^{2 n+1}\right)$, then $H^{\prime}(p x)=0$, since, by [6], $H^{\prime}$ factors through the mod $p$ reduction of the cobar complex. Thus we need merely note that $h_{1}^{j(p-1)} h_{2} v_{1}^{j-1} h_{1} l_{3}, p^{p-2} h_{1}^{j(p-1)+p} v_{1}^{j} h_{1} l_{3}$, and $p^{i-1} h_{1}^{j(p-1)+i} v_{1}^{j-1} h_{1} l_{3}$ are defined, using 2.6(ii) to replace $p h_{1}$.

Now we settle the extension questions in Theorems 2.4 and 2.5.

Theorem 2.23. $E_{2}^{1,2 n+q m+1}(B(2 n+1,2 n+q+1)), E_{2}^{1, q(k+m)+3}\left(B_{k}(3, k q+3)\right)$ in Theorems 2.4 and 2.5 are cyclic groups.

Proof. We begin with Theorem 2.4. We will show that whenever $\operatorname{ker}(\partial) \neq$ 0 in the exact sequence of 2.4 , there is an element $z \in E_{2}^{1, t}(B)$ such that $j_{*}(z)=\alpha_{m-1} l_{2 n+q+1}$, the element of order $p$, and $p z=i_{*}$ (gen). Since $\partial\left(\alpha_{m-1} l_{2 n+q+1}\right)=0$ in these cases, there is $w \in E_{2}^{1,2 n+q m+1}\left(S^{2 n+1}\right)$ such that $d(w)=\alpha_{m-1} \alpha_{1} l_{2 n+1}$. Let

$$
z=\alpha_{m-1} l_{2 n+q+1}-w l_{2 n+1} .
$$


Then $z$ is a cycle, since $d(z)=\alpha_{m-1} \alpha_{1} l_{2 n+1}-\alpha_{m-1} \alpha_{1} l_{2 n+1}$, and clearly $j_{*}(z)$ is as required. Since, by $2.9, p \alpha_{m-1}=d\left(v_{1}^{m-1}\right)$, we have

$$
\begin{aligned}
p z-d\left(v_{1}^{m-1} l_{2 n+q+1}\right)= & d\left(v_{1}^{m-1}\right) l_{2 n+q+1}-p w l_{2 n+1} \\
& -d\left(v_{1}^{m-1}\right) l_{2 n+q+1}+v_{1}^{m-1} \alpha_{1} l_{2 n+1} \\
= & i_{*}\left(\left(v_{1}^{m-1} \alpha_{1}-p w\right) l_{2 n+1}\right) .
\end{aligned}
$$

We consider the morphism of $E_{2}$-terms

$$
E_{2}^{1}\left(S^{2 n+1}\right) \rightarrow E_{2}^{1}\left(S^{0}\right) \rightarrow E_{2}^{1}(M)
$$

obtained by stabilizing the cobar complex and reducing it mod $p$. The image of $\left(v_{1}^{m-1} \alpha_{1}-p w\right) l_{2 n+1}$ is $v_{1}^{m-1} h_{1}$, which is nonzero by Lemma 2.11. Since $E_{2}^{1,2 n+q m+1}\left(S^{2 n+1}\right)$ is a cyclic group, $\left(v_{1}^{m-1} \alpha_{1}-p w\right) l_{2 n+1}$ must then be a generator, since it maps nontrivially to a $\mathbf{Z} / p$.

The argument for Theorem 2.5 works similarly up to the point of showing that $\left(v_{1}^{m} \alpha_{k}-p w\right) l_{3} \neq 0 \in E_{2}^{1, q(k+m)+3}\left(S^{3}\right)$. We show this by noting from [6, $\S 7]$ that the Hopf invariant

$$
H_{2}: E_{2}^{1}\left(S^{3}\right) \rightarrow \operatorname{Ext}^{0}(W(1))
$$

factors through the mod $p$ reduction of the unstable cobar complex. Thus

$$
H_{2}\left(\left(v_{1}^{m} \alpha_{k}-p w\right) l_{3}\right)=H_{2}\left(v_{1}^{m+k} h_{1} l_{3}\right)=v_{1}^{m+k} \neq 0 .
$$

The first " $=$ " uses [7, p. 246] to express $\alpha_{k} \bmod S^{1}$, the second uses [4, 5.3], and the " $\neq$ " uses $[2,3.4]$.

We close this section by proving the following result mentioned earlier, which completes the proof of Theorem 2.1.

Theorem 2.24. In Theorem 2.1, the group $v_{1}^{-1} \pi_{q(k+m)+1}\left(B_{k}(3, k q+3)\right)$ is cyclic. Proof. We use the exact sequence in $v_{1}^{-1} \pi_{*}(-)$ of the fibration of Theorem 2.1. Since

$$
v_{1}^{-1} \pi_{q(k+m)+1}\left(S^{3}\right) \approx \mathbf{Z} / p
$$

the cyclicity follows from that of $v_{1}^{-1} \pi_{q(k+m)+1}\left(S^{k q+3}\right)$ unless

$$
\partial=0: v_{1}^{-1} \pi_{q(k+m)+2}\left(S^{k q+3}\right) \rightarrow v_{1}^{-1} \pi_{q(k+m)+1}\left(S^{3}\right) .
$$

If $(2.25)$ is satisfied, then

$$
\circ \alpha_{1} \neq 0: v_{1}^{-1} \pi_{q(k+m-1)+2}\left(S^{k q+3}\right) \rightarrow v_{1}^{-1} \pi_{q(k+m)+1}\left(S^{k q+3}\right)
$$

by $[13,6.2]$, and

$$
\partial: v_{1}^{-1} \pi_{q(k+m-1)+2}\left(S^{k q+3}\right) \rightarrow v_{1}^{-1} \pi_{q(k+m-1)+1}\left(S^{3}\right)
$$

is an isomorphism of $\mathbf{Z} / p$ 's by Theorem 2.5. Let $G$ denote a generator of $v_{1}^{-1} \pi_{q(k+m-1)+2}\left(S^{k q+3}\right)$, and let

$$
Y \in v_{1}^{-1} \pi_{q(k+m)+1}(B(3, k q+3))
$$

project to $G \circ \alpha_{1}$. By [22, 2.1],

$$
p Y=i_{*}\left(\left\langle\partial G, \alpha_{1}, p\right\rangle\right)=\partial(G) \circ v_{1} \neq 0 .
$$




\section{3. $v_{1}$-PERIODIC HOMOTOPY GROUPS OF A COMPLEX STIEFEL MANIFOLD}

In this section we prove Theorem 1.4. The bulk of the work, by far, is the last case, which amounts to calculating certain $v_{1}$-periodic homotopy groups of the complex Stiefel manifold $\mathrm{SU}(30) / \mathrm{SU}(11)$.

By the definition of $B(\cdots)$ given in Theorem 1.1, the first four cases of Theorem 1.4 amount to just giving explicit values for $v_{1}^{-1} \pi_{*}(\mathrm{SU}(n))$, for certain small values of $n$. It was proved in [13] that $v_{1}^{-1} \pi_{2 k}(\mathrm{SU}(n)) \approx \mathrm{Z} / p^{e_{p}(k, n)}$, where $e_{p}(k, n)$ is the minimal $p$-exponent in a set of numbers related to Stirling numbers of the second kind. These numbers had been studied earlier in [12], where it was shown that for fixed $n$ they are periodic in $k$, so that a finite number of calculations suffice to determine $e_{p}(k, n)$ for all $k$. The first four cases of Theorem 1.4 are a consequence of these remarks and extensive computer calculations performed by the second author using Mathematica.

In the remainder of this section we prove the last case of Theorem 1.4. One might be inclined to think, as we initially were, that the result follows from the exact sequence in $v_{1}^{-1} \pi_{*}(-)$ associated to the fibration

$$
S^{11} \rightarrow \mathrm{SU}^{\prime}(30) \rightarrow B(23,35,47,59),
$$

where $\operatorname{SU}^{\prime}(30)=B(11,23,35,47,59)$ is a direct factor of $\operatorname{SU}(30)$, so that $v_{1}^{-1} \pi_{*}\left(\mathrm{SU}^{\prime}(30)\right)$ is known by [13]. However, we do not have a priori knowledge of the morphism

$$
v_{1}^{-1} \pi_{2 k-1}\left(S^{11}\right) \rightarrow v_{1}^{-1} \pi_{2 k-1}\left(\mathrm{SU}^{\prime}(30)\right)
$$

Diagram chasing applied to the many fibrations involving subquotients of $\mathrm{SU}^{\prime}(30)$ will, with the known results for $\mathrm{SU}^{\prime}(n)$ 's, spheres, and $B(n, n+12)$ 's, yield results for some, but not all, of the groups $v_{1}^{-1} \pi_{2 k}(B(23,35,47,59))$.

Instead of working our way down from $\mathrm{SU}^{\prime}(30)$, we work our way up, one sphere at a time. We begin by extracting the following result from Theorem 1.3(2). Throughout the remainder of the paper, $p=7, \nu(-)=\nu_{7}(-)$, and $\equiv$ is $\bmod 7$.

Corollary 3.1. The groups whose orders are listed below are cyclic 7-groups.

$$
\begin{aligned}
& \nu\left(\left|v_{1}^{-1} \pi_{12 m+22}(B(23,35))\right|\right)= \begin{cases}\min (11,2+\nu(m)), & m \neq 1, \\
\min (17,2+\nu(m-1)), & m \equiv 1,\end{cases} \\
& \nu\left(\left|v_{1}^{-1} \pi_{12 m+22}(B(35,47))\right|\right)= \begin{cases}\min (17,2+\nu(m-1)), & m \neq \equiv 2, \\
\min (23,2+\nu(m-2)), & m \equiv 2,\end{cases} \\
& \nu\left(\left|v_{1}^{-1} \pi_{12 m+22}(B(47,59))\right|\right)= \begin{cases}\min (23,2+\nu(m-2)), & m \neq \equiv 3, \\
\min (29,2+\nu(m-3)), & m \equiv 3 .\end{cases}
\end{aligned}
$$

We will use this result and a delicate UNSS analysis to deduce the following result, from which the desired result, the last case of 1.4 , will follow by another UNSS analysis.

Proposition 3.2. The groups whose orders are listed below are cyclic 7-groups.

$$
\nu\left(\left|v_{1}^{-1} \pi_{12 m+22}(B(23,35,47))\right|\right)= \begin{cases}\min (11,3+\nu(m)), & m \neq 1,2, \\ \min (17,3+\nu(m-1)), & m \equiv 1, \\ \min (23,3+\nu(m-2)), & m \equiv 2,\end{cases}
$$




$$
\nu\left(\left|v_{1}^{-1} \pi_{12 m+22}(B(35,47,59))\right|\right)= \begin{cases}\min (17,3+\nu(m-1)), & m \neq 2,3, \\ \min (23,3+\nu(m-2)), & m \equiv 2, \\ \min (29,3+\nu(m-3)), & m \equiv 3\end{cases}
$$

We will prove the second part of Proposition 3.2; the proof of the first part is completely analogous. We will abbreviate $v_{1}^{-1} \pi_{*}(B(\bar{n}))$ to $v_{*}(\bar{n})$. In particular, $v_{*}(n)$ denotes $v_{1}^{-1} \pi_{*}\left(S^{n}\right)$. Where convenient, we use $2 k=12 m+22$. We will use the following commutative diagram of exact sequences:

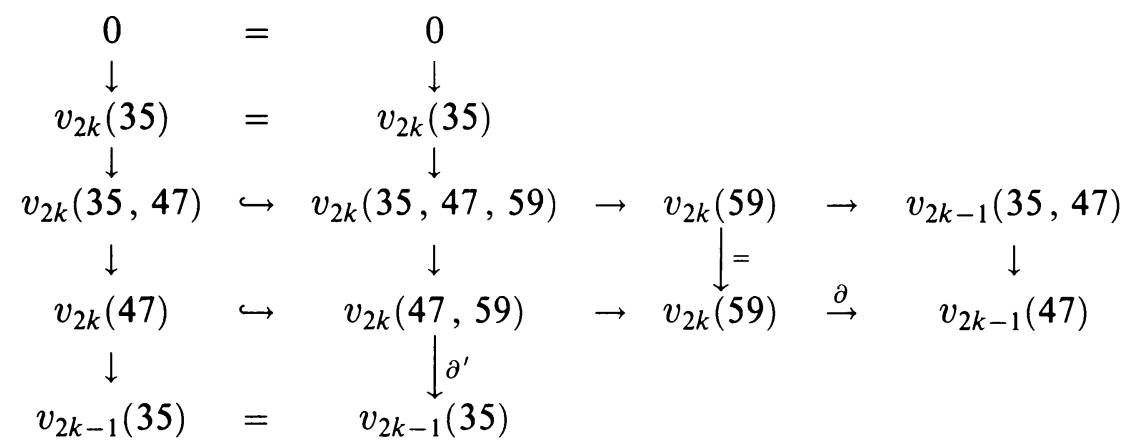

All groups are known cyclic groups except the desired group $v_{2 k}(35,47,59)$ and $v_{2 k-1}(35,47)$, which might not be cyclic, but does not enter into the argument. The crux is the determination of $\partial^{\prime}$. This yields the order of $v_{2 k}(35,47,59)$, and its cyclicity follows since the element of order $p$ in $v_{2 k}(47,59)$ comes from that of $v_{2 k}(47)$, which extends to the generator of $v_{2 k}(35)$.

For example, in the second case of the table below, our earlier results tell us that the exact sequence in the second column of the above diagram is

$$
0 \rightarrow \mathbf{Z} / p^{16} \rightarrow ? \rightarrow \mathbf{Z} / p^{2} \stackrel{\partial^{\prime}}{\rightarrow} \mathbf{Z} / p
$$

and the UNSS analysis below shows that $\partial^{\prime}$ is nonzero. Hence the unknown group has order $p^{17}$, and it must be cyclic because in (3.3) the horizontal map to it is an injection from $\mathrm{Z} / p^{17}$.

All groups in the table below are cyclic 7-groups, and all but the last column are known from our earlier work.

\begin{tabular}{c|ccccc} 
& $\left|v_{2 k}(59)\right|$ & $\left|v_{2 k-1}(47)\right|$ & $\left|v_{2 k}(47,59)\right|$ & $\left|v_{2 k-1}(35)\right|$ & $\left|\operatorname{im}\left(\partial^{\prime}\right)\right|$ \\
\hline$m \not \equiv 2,3 ; \quad \nu(m-1)=t \leq 14$ & $p$ & $p$ & $p^{2}$ & $p^{t+1}$ & 0 \\
$\nu(m-1)=15$ & $p$ & $p$ & $p^{2}$ & $p^{16}$ & $p$ \\
$\nu(m-1) \geq 16$ & $p$ & $p$ & $p^{2}$ & $p^{17}$ & $p^{2}$ \\
$1 \leq \nu(m-2)=t \leq 20$ & $p$ & $p^{t+1}$ & $p^{t+2}$ & $p$ & 0 \\
$\nu(m-2)=21$ & $p$ & $p^{22}$ & $p^{23}$ & $p$ & $p$ \\
$\nu(m-2) \geq 22$ & $p$ & $p^{23}$ & $p^{23}$ & $p$ & $p$ \\
$1 \leq \nu(m-3)=t \leq 26$ & $p^{t+1}$ & $p$ & $p^{t+2}$ & $p$ & 0 \\
$\nu(m-3)=27$ & $p^{28}$ & $p$ & $p^{29}$ & $p$ & $p$ \\
$\nu(m-3) \geq 28$ & $p^{29}$ & $p$ & $p^{29}$ & $p$ & $p$
\end{tabular}

Now we must establish that $\left|\operatorname{im}\left(\partial^{\prime}\right)\right|$ is as claimed in the table. The result in the sixth case follows by diagram chasing, but aside from that we work in the UNSS as in $\S 2$, so that $v_{2 k}(-) \approx E_{2}^{1,2 k+1}(-)$ and $v_{2 k-1}\left(S^{n}\right) \approx \widetilde{E}_{2}^{2,2 k+1}\left(S^{n}\right)$. We begin by noting that if $y l_{59}$ generates $\operatorname{ker}(\partial)$, and if, letting $d$ denote the boundary in the unstable cobar complex,

$$
\partial\left(y l_{59}\right)=y \alpha_{1} l_{47}=d\left(w l_{47}\right),
$$


then $y x_{59}-w x_{47}$ generates $E_{2}^{1,2 k+1}(47,59)$, and

$$
\partial^{\prime}\left(y x_{59}-w x_{47}\right)=y \otimes\left(136 h_{1}^{2}-h_{1} v_{1}\right) l_{35}-w \otimes 17 h_{1} l_{35}
$$

Here we use [3, 3.4] for the coaction in SU, which determines the boundary morphisms in the exact sequences obtained when building it up, and the explicit formula (recall $p=7$ )

$$
\sum^{F^{*}} h_{s}=1+h_{1}+h_{1} v_{1}+h_{1} v_{1}^{2}-3 h_{1}^{2} v_{1}+\text { higher terms }
$$

which can be derived as in $[9,2.8]$. The 136 is obtained as $\left(\begin{array}{c}17 \\ 2\end{array}\right)$.

By Proposition 2.9, $y$ will be $\alpha_{m-3}$ in the first six cases of the table, and it will be $\alpha_{m-3 / \min (t+1,28)}$ in the last three. In the first three cases, $w=v_{1}^{m-4} h_{1}^{2}$ by $2.12(3)(a)$, since we are in a stem where the unstable towers have order $p$. Thus

$$
\partial^{\prime}(\text { gen })=\left(\alpha_{m-3}\left(136 h_{1}^{2}-h_{1} v_{1}\right)-v_{1}^{m-4} h_{1}^{2} \otimes 17 h_{1}\right) l_{35},
$$

and by (2.7) and (2.10) this desuspends to $S^{5}$, where its first term establishes it as a generator by $2.12(3)(\mathrm{b})$. Now establishing the claim about $\operatorname{im}\left(\partial^{\prime}\right)$ in the first three cases just amounts to noting that by 2.12(1) the iterated suspension

$$
E_{2}^{2, q(m-1)+5}\left(S^{5}\right) \rightarrow E_{2}^{2, q(m-1)+35}\left(S^{35}\right)
$$

is 0 if $t<15$, has image of order $p$ if $t=15$, and is injective if $t \geq 16$.

In the fourth and fifth cases, $w=v_{1}^{m-t-4} h_{1}^{t+2}$ by $2.12(3)(\mathrm{a})$. This time

$$
\partial^{\prime}(\text { gen })=\left(\alpha_{m-3}\left(136 h_{1}^{2}-h_{1} v_{1}\right)-v_{1}^{m-t-4} h_{1}^{t+2} \otimes 17 h_{1}\right) l_{35}
$$

lies in a $\mathbf{Z} / p$, and so is zero if it double desuspends. By (2.10), the first part desuspends to $S^{5}$, and by (2.7) $v_{1}^{m-t-4} h_{1}^{t+2} \otimes 17 h_{1} l_{35}$ double desuspends if $t+2 \leq 22$. If $t=21$, Corollary 2.15 implies that $v_{1}^{m-24} h_{1}^{23} \otimes h_{1} l_{35} \neq 0$.

In the seventh case with $t>5$ and the eighth case, $w=v_{1}^{m-t+2} h_{1}^{t-4}$. To see this, we note that by (2.10) $\alpha_{m-3 / t+1} \alpha_{1} l_{47}$ desuspends to $\alpha_{m-3 / t+1} \alpha_{1} l_{2 t-9}$, where it is the element of order $p$ by 2.15 , and hence is $d\left(v_{1}^{m-t+2} h_{1}^{t-4} l_{47}\right)$ by $2.12(3)(a)$. Now, in the seventh case with $t>5$,

$$
\partial^{\prime}(\text { gen })=\left(\alpha_{m-3 / t+1}\left(136 h_{1}^{2}-h_{1} v_{1}\right)-v_{1}^{m-t+2} h_{1}^{t-4} \otimes 17 h_{1}\right) l_{35}
$$

lies in a $\mathbf{Z} / p$. The first part desuspends by (2.10), as does the second since $t-4 \leq 22$, and so $\partial^{\prime}$ (gen) $=0$. The eighth case is similar except we do not have $t-4 \leq 22$. Here, mod terms that desuspend, $\partial^{\prime}($ gen $)=17 v_{1}^{m-24} h_{1}^{23} \otimes h_{1} l_{29} \neq 0$ by 2.15 as above. The ninth case, like the eighth, has $y=\alpha_{m-3 / 22}$ and $w=$ $v_{1}^{m-24} h_{1}^{23} l$, and so is completed just like the eighth case. In the seventh case with $t \leq 5, \alpha_{m-3 / t+1} \otimes \alpha_{1} l_{47}$ desuspends to $S^{1}$ by (2.10), and hence so does $w$. Thus the term $w \otimes 17 h_{1} l_{35}$ in $\partial^{\prime}$ (gen) double desuspends and hence is 0 , completing the proof of Proposition 3.2.

Now we are ready to prove the last case of Theorem 1.4. Although the proof is very similar to the one we just finished, it is a bit more complicated because of the extra cell, and so we write out the details. We will use the following 
commutative diagram of exact sequences:

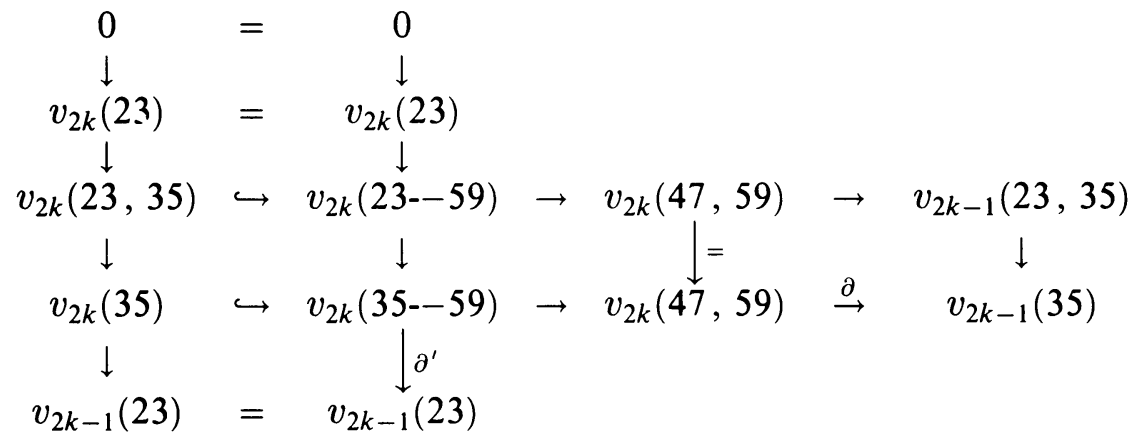

Note that $(23,35,47,59)$ has been abbreviated to $(23-59)$, and $(35,47$, 59) similarly. As before, we seek to determine $\partial^{\prime}$, which, with the other known cyclic groups, will tell the order of $v_{2 k}$ (23-59), and its cyclicity follows from the diagram. Again we divide into cases, where all groups except the last column are known cyclic groups, and the last column will be established by UNSS analysis.

\begin{tabular}{c|ccccc} 
& $\left|v_{2 k}(47,59)\right|$ & $\left|v_{2 k-1}(35)\right|$ & $\left|v_{2 k}(35-59)\right|$ & $\left|v_{2 k-1}(23)\right|$ & $\left|\mathrm{im}\left(\partial^{\prime}\right)\right|$ \\
\hline$m \neq 1,2,3 ; \nu(m)=t \leq 7$ & $p^{2}$ & $p$ & $p^{3}$ & $p^{t+1}$ & 0 \\
$\nu(m)=8$ & $p^{2}$ & $p$ & $p^{3}$ & $p^{9}$ & $p$ \\
$\nu(m)=9$ & $p^{2}$ & $p$ & $p^{3}$ & $p^{10}$ & $p^{2}$ \\
$\nu(m) \geq 10$ & $p^{2}$ & $p$ & $p^{3}$ & $p^{11}$ & $p^{3}$ \\
\hline $1 \leq \nu(m-1)=t \leq 13$ & $p^{2}$ & $p^{t+1}$ & $p^{t+3}$ & $p$ & 0 \\
$\nu(m-1)=14$ & $p^{2}$ & $p^{15}$ & $p^{17}$ & $p$ & $p$ \\
$\nu(m-1)=15$ & $p^{2}$ & $p^{16}$ & $p^{17}$ & $p$ & $p$ \\
$\nu(m-1) \geq 16$ & $p^{2}$ & $p^{17}$ & $p^{17}$ & $p$ & $p$ \\
\hline $1 \leq \nu(m-2)=t \leq 19$ & $p^{t+2}$ & $p$ & $p^{t+3}$ & $p$ & 0 \\
$\nu(m-2)=20$ & $p^{22}$ & $p$ & $p^{23}$ & $p$ & $p$ \\
$\nu(m-2) \geq 21$ & $p^{23}$ & $p$ & $p^{23}$ & $p$ & $p$ \\
\hline $1 \leq \nu(m-3)=t \leq 25$ & $p^{t+2}$ & $p$ & $p^{t+3}$ & $p$ & 0 \\
$\nu(m-3)=26$ & $p^{28}$ & $p$ & $p^{29}$ & $p$ & $p$ \\
$\nu(m-3) \geq 27$ & $p^{29}$ & $p$ & $p^{29}$ & $p$ & $p$
\end{tabular}

The argument proceeds very similarly to the previous one. The boundary morphisms are determined by

$$
\begin{aligned}
\psi\left(x_{59}\right)= & 1 \otimes x_{59}+23 h_{1} \otimes x_{47}+\left(\left(\begin{array}{c}
17 \\
2
\end{array}\right) h_{1}^{2}-17 h_{1} v_{1}\right) x_{35} \\
& +\left(\left(\begin{array}{c}
11 \\
3
\end{array}\right) h_{1}^{3}-143 h_{1}^{2} v_{1}+11 h_{1} v_{1}^{2}\right) x_{23} .
\end{aligned}
$$

As in the previous argument, the coefficients (which are units) do not play a role, as there is always one term which dominates, and so there is no possibility of cancelling, and so we will ignore coefficients. Also, in the formulas for $\psi(x)$, the terms which have one or more $v_{1}$ 's on the right desuspend farther than the $h_{1}^{e}$-terms, and so may be omitted from the analysis. We will adopt these conventions.

In the first block of four cases, the above proof of Proposition 3.2 shows that the generator of $E_{2}^{1,2 k+1}(47,59)$ is $\alpha_{m-3} x_{59}-v_{1}^{m-4} h_{1}^{2} x_{47}$, and

$$
\partial(\text { gen })=\left(\alpha_{m-3} h_{1}^{2}-v_{1}^{m-4} h_{1}^{2} \otimes h_{1}\right) x_{35} .
$$


By 2.12(3), this equals $d\left(v_{1}^{m-4} h_{1}^{3} x_{35}\right)$, and so, with the conventions mentioned above, the generator of $E_{2}^{1,2 k+1}(35-59)$ is $\alpha_{m-3} x_{59}+v_{1}^{m-4} h_{1}^{2} x_{47}+v_{1}^{m-4} h_{1}^{3} x_{35}$. Therefore,

$$
\partial^{\prime}(\text { gen })=\left(\alpha_{m-3} \otimes h_{1}^{3}+v_{1}^{m-4} h_{1}^{2} \otimes h_{1}^{2}+v_{1}^{m-4} h_{1}^{3} \otimes h_{1}\right) x_{23} .
$$

This desuspends to the generator on $S^{7}$ (due to the first term, by 2.12(3)(c)), and the result follows from the result of 2.12(1) that

$$
\left|\operatorname{im}\left(E_{2}^{2, q m+7}\left(S^{7}\right) \rightarrow E_{2}^{2, q m+23}\left(S^{23}\right)\right)\right|= \begin{cases}0, & \nu(m) \leq 7, \\ p, & \nu(m)=8, \\ p^{2}, & \nu(m)=9, \\ p^{3}, & \nu(m) \geq 10 .\end{cases}
$$

In the second block of four cases, the $\partial$ of the generator of $(47,59)$ is as in the previous block, with $\alpha_{m-3} \otimes h_{1}^{2} x_{35}$ as its predominant term. By 2.12(3)(b), this is $d\left(v_{1}^{m-t-4} h_{1}^{t+3} l_{35}\right)$ in the first two cases of this block. Hence in these cases the generator of $E_{2}^{1,2 k+1}(35-59)$ is $\alpha_{m-3} x_{59}+v_{1}^{m-4} h_{1}^{2} x_{47}+v_{1}^{m-t-4} h_{1}^{t+3} x_{35}$, and

$$
\partial^{\prime}(\text { gen })=\left(\alpha_{m-3} \otimes h_{1}^{3}+v_{1}^{m-4} h_{1}^{2} \otimes h_{1}^{2}+v_{1}^{m-t-4} h_{1}^{t+3} \otimes h_{1}\right) l_{23}
$$

in a stem where the groups have order $p$. By (2.7), this desuspends (and hence is 0 ) if $t+3 \leq 16$, while by 2.15 it is nonzero if $t=14$. In the third case in this second block, $\operatorname{ker}(\partial)$ is generated by $p$ times the generator of $E_{2}^{1,2 k+1}(47,59)$. Replacing $p h_{1}$ by $v_{1}$ (using 2.6 and noting that $\eta\left(v_{1}\right)$ can be ignored since it will desuspend farther), we can write the generator of $\operatorname{ker}(\partial)$ in this case as $v_{1}^{m-3} h_{1} x_{47}+v_{1}^{m-18} h_{1}^{17} x_{35}$, which $\partial^{\prime}$ sends to $h_{1}^{17} \otimes h_{1} x_{23} \neq 0$. The fourth case in this second block has $\operatorname{ker}(\partial)=0$, and so the second column of $(3.5)$ is isomorphic to the first column, which is known. is

Now for the third block: By the proof of 3.2, the generator of $E_{2}^{1,2 k+1}(47,59)$

$$
\begin{cases}\alpha_{m-3} x_{59}+v_{1}^{m-t-4} h_{1}^{t+2} x_{47} & \text { if } t \leq 21 \\ \alpha_{m-2 / 23} x_{47} & \text { if } t>21\end{cases}
$$

The generator of $\operatorname{ker}(\partial)$ is

$$
\begin{cases}\alpha_{m-3} x_{59}+v_{1}^{m-t-4} h_{1}^{t+2} x_{47} & \text { if } t \leq 20, \\ d\left(v_{1}^{m-3}\right) x_{59}+v_{1}^{m-24} h_{1}^{22} x_{47} & \text { if } t=21, \\ \alpha_{m-2 / 22} x_{47} & \text { if } t>21,\end{cases}
$$

which, by $2.12(3)$, maps by $\partial$ to $d\left(v_{1}^{m-1-e} h_{1}^{e} l_{35}\right)$, where $e=. . \mathrm{in}(t-3,17)$. In verifying this, remember that $\partial$ effectively just replaces $x_{59}$ by $h_{1}^{2} x_{35}$, and $x_{47}$ by $h_{1} x_{35}$. Therefore, the generator of $E_{2}^{1,2 k+1}(35-59)$ is obtained by adding $v_{1}^{m-1-e} h_{1}^{e} l_{35}$ to (3.6). Mod terms which desuspend farther, $\partial^{\prime}($ gen $)=$ $v_{1}^{m-1-e} h_{1}^{e} \otimes h_{1} l_{23}$, and this is 0 if $t-3<20$, and nonzero if $t-3 \geq 20$, as claimed.

Finally, we handle the fourth block by a similar argument. If $t \leq 5$, the slightly special considerations required in the seventh case of the proof of 3.2 are required; they only make things easier, and so we assume $t>5$. The generator of $E_{2}^{1,2 k+1}(47,59)$ is

$$
\begin{cases}\alpha_{m-3 / t+1} x_{59}+v_{1}^{m-t+2} h_{1}^{t-4} x_{47} & \text { if } t<27 \\ \alpha_{m-3 / 28} x_{59}+v_{1}^{m-25} h_{1}^{23} x_{47} & \text { if } t \geq 27\end{cases}
$$


and the generator maps nontrivially under $\partial$ if $t \geq 27$. Thus, if $e=$ $\min (t+1,27)$, the generator of $\operatorname{ker}(\partial)$ is

$$
\alpha_{m-3 / e} x_{59}+v_{1}^{m-e+3} h_{1}^{e-5} x_{47} .
$$

Here we have again used $p h_{1}=v_{1}$ mod terms that desuspend farther. The image under $\partial$ of $(3.7)$ is $d\left(v_{1}^{m+9-e} h_{1}^{e-10}{ }_{35}\right)$ by 2.12(3) applied to the second term. Note that the first term double desuspends one step farther, so that if 2.12(3) was applied to it, we would obtain $v_{1}^{m+10-e} h_{1}^{e-11}=p v_{1}^{m+9-e} h_{1}^{e-10}$. Hence, the generator of $E_{2}^{1,2 k+1}(35-59)$ is

$$
\alpha_{m-3 / e} x_{59}+v_{1}^{m-e+3} h_{1}^{e-5} x_{47}+v_{1}^{m-e+9} h_{1}^{e-10} x_{35},
$$

and

$$
\partial^{\prime}(\text { gen })=\left(v_{1}^{m-e-3} h_{1}^{e} \otimes h_{1}^{3}+v_{1}^{m-e+3} h_{1}^{e-5} \otimes h_{1}^{2}+v_{1}^{m-e+9} h_{1}^{e-10} \otimes h_{1}\right) l_{23},
$$

and, as usual, this is 0 if $e<27$ and nonzero if $e=27$, with the last term the dominant one.

\section{REFERENCES}

1. J. F. Adams, On the groups $J(X)$. IV, Topology 5 (1966), 21-71.

2. M. Bendersky, Unstable towers in the odd primary homotopy groups of spheres, Trans. Amer. Math. Soc. 287 (1985), 529-542.

3. $\_$, Some calculations in the unstable Adams-Novikov spectral sequence, Publ. Res. Inst. Math. Sci. 16 (1980), 739-766.

4. 276 (1983), 599-619.

5. __ The $v_{1}$-periodic unstable Novikov spectral sequence, Topology (to appear).

6. _ The BP Hopf invariant, Amer. J. Math. 108 (1986), 1037-1058.

7. M. Bendersky, E. B. Curtis, and H. R. Miller, The unstable Adams spectral sequence for a generalized homology, Topology 17 (1978), 229-248.

8. M. Bendersky and D. M. Davis, 2-primary $v_{1}$-periodic homotopy groups of $S U(n)$, Amer. J. Math. (to appear).

9. __ Unstable BP-homology and desuspensions, Amer. J. Math. 107 (1985), 833-852.

10. A. Borel, Sous-groupes commutatifs et torsion des groupes compact connexes, Tôhoku J. Math. 13 (1961), 216-240.

11. F. R. Cohen, J. C. Moore, and J. A. Neisendorfer, The double suspension and exponents of the homotopy groups of spheres, Ann. of Math. (2) 110 (1979), 549-565.

12. M. Crabb and K. Knapp, The Hurewicz map on stunted complex projective spaces, Amer. J. Math. 110 (1988), 783-809.

13. D. M. Davis, $v_{1}$-periodic homotopy groups of $S U(n)$ at an odd prime, J. London Math. Soc. (to appear).

14. D. M. Davis and M. Mahowald, Three contributions to the homotopy theory of the exceptional Lie groups $G_{2}$ and $F_{4}$, J. Math. Soc. Japan (to appear).

15. $\ldots, v_{1}$-periodicity in the unstable Adams spectral sequence, Math. Z. 204 (1990), 319339.

16. Math. Soc. Lecture Notes (to appear).

17. I. M. James, On Lie groups and their homotopy groups, Proc. Cambridge Phil. Soc. 55 (1959), 244-247.

18. A. T. Lundell, A divisibility property for Stirling numbers, J. Number Theory 10 (1978), $35-54$. 
19. M. Mahowald, The image of $J$ in the EHP sequence, Ann. of Math. (2) 116 (1982), 65-112.

20. H. R. Miller and D. C. Ravenel, Morava stabilizer algebras and the localization of Novikov's $E_{2}$-term, Duke Math. J. 44 (1977), 433-447.

21. M. Mimura, G. Nishida, and H. Toda, Mod p decomposition of compact Lie groups, Publ. Res. Inst. Math. Sci. 13 (1977), 627-680.

22. M. Mimura and H. Toda, Homotopy groups of $S U(3), S U(4)$, and $S p(2)$, J. Math. Kyoto Univ. 3 (1964), 217-250.

23. Cohomology operations and the homotopy of compact Lie groups. I, Topology 9 (1970), 317-336.

24. D. C. Ravenel, Complex cobordism and the stable homotopy groups of spheres, Academic Press, 1986.

25. P. Selick, Moore conjectures, Lecture Notes in Math., vol. 1318, Springer-Verlag, 1988, pp. 219-227.

26. R. D. Thompson, The $v_{1}$-periodic homotopy groups of an unstable sphere at odd primes, Trans. Amer. Math. Soc. 319 (1990), 535-560.

27. C. Wilkerson, Self-maps of classifying spaces, Lecture Notes in Math., vol. 418, SpringerVerlag, 1974, pp. 150-157.

Department of Mathematics, CUNy, Hunter College, New York, New York 10021

Department of Mathematics, Lehigh University, Bethlehem, Pennsylvania 18015

Department of Mathematics, Okayama University, Okayama 707, Japan 